\title{
REPRESENTATIONS OF LIE ALGEBRAS BY NORMAL OPERATORS
}

THOMAS SHERMAN ${ }^{1}$

Recently I. E. Segal [2] has proposed a study of the unitary representations of a complex semisimple Lie group $G$ by studying "analytic" holomorphic representations of $G$ by normal operators. To this end he proved that every unitary representation $U$ of $G$ may be written $U(g)=R(g) R\left(g^{-1}\right)^{*}(g \in G)$ where $R$ is an analytic holomorphic representation of $G$ by normal operators such that if $R\left(g_{1}\right)$ and $R\left(g_{2}\right)$ are defined then $R\left(g_{1}\right)$ commutes with $R\left(g_{2}\right)^{*}$.

Now a representation of a group by normal operators is a peculiar phenomenon because the product of two normal operators is usually not normal. In this paper we study this peculiarity in Lie algebraic terms. We achieve a decomposition of a representation of a semisimple Lie algebra by normal operators into the sum of two representations which commute with each other. One of these is by skewadjoint operators, and the other is a representation (necessarily by normal operators) which commutes with its contragredient.

We begin by establishing our results in the following generalised setting: Let $\mathfrak{u}$ be a Lie algebra over some fixed field of characteristic other than 2. On $\mathfrak{u}$ we assume the existence of a linear mapping sending an element $x$ of $\mathfrak{u}$ into $x^{*}$ such that $\left(x^{*}\right)^{*}=x$ and $\left[x^{*}, y^{*}\right]$ $=-[x, y]^{*}$. In other words our mapping is an anti-automorphism of order 2. If $\mathfrak{a}$ and $\mathfrak{b}$ are subsets of $\mathfrak{u}$ let $[\mathfrak{a}, \mathfrak{b}]=$ the linear span of $\{[x, y] \mid x \in \mathfrak{a}, y \in \mathfrak{b}\}$ and $\mathfrak{a}^{*}=\left\{x^{*} \mid x \in \mathfrak{a}\right\}$. We will say that an element $x$ of $\mathfrak{u}$ is $n r m l$ if $\left[x, x^{*}\right]=0$ and that $x$ is skew if $x^{*}=-x$.

Lemma. Let $\mathfrak{g}$ be a subalgebra of $\mathfrak{u}$ consisting of nrml elements. Then $\mathfrak{g}^{+}=\mathfrak{g}+\mathfrak{g}^{*}+\left[\mathfrak{g}, \mathfrak{g}^{*}\right]$ is a Lie algebra and $\mathfrak{i}=\left[\mathfrak{g}, \mathfrak{g}^{*}\right]$ is an ideal in $\mathfrak{g}^{+}$ consisting of skew elements.

Proof. For any $x$ and $y$ in $g$ we have

$$
\begin{aligned}
0 & =\left[x+y,(x+y)^{*}\right]=\left[x, x^{*}\right]+\left[x, y^{*}\right]+\left[y, x^{*}\right]+\left[y, y^{*} 1\right. \\
& =\left[x, y^{*}\right]+\left[y, x^{*}\right] .
\end{aligned}
$$

But $\left[y, x^{*}\right]=-\left[y^{*}, x\right]^{*}$ so $\left[x, y^{*}\right]$ is skew. Since every element of $\left[\mathfrak{g}, \mathfrak{g}^{*}\right]$ is a linear combination of such elements we have at least shown that $\left[g ; g^{*}\right]$ consists entirely of skew elements. To prove that $i=\left[g, g^{*}\right]$

Received by the editors June 3, 1964 and, in revised form, September 14, 1964.

1 This research was supported in part by the Woodrow Wilson Foundation. 
is an ideal, we have only to show that $[\mathfrak{g}, \mathfrak{i}] \subseteq \mathfrak{i}$. For then $\left[\mathfrak{g}^{*}, \mathfrak{i}\right]$ $=[\mathfrak{g}, \mathfrak{i}]^{*} \subseteq \mathfrak{i}^{*}=\mathfrak{i}$ and

$$
[\mathfrak{i}, \mathfrak{i}]=\left[\left[\mathfrak{g}, \mathfrak{g}^{*}\right], \mathfrak{i}\right] \subseteq\left[[\mathfrak{g}, \mathfrak{i}], \mathfrak{g}^{*}\right]+\left[\mathfrak{g},\left[\mathfrak{g}^{*}, \mathfrak{t}\right]\right] \subseteq\left[\mathfrak{g}^{*}, \mathfrak{i}\right]+[\mathfrak{g}, \mathfrak{i}] \subseteq \mathfrak{i} .
$$

So $\mathfrak{g}+\mathfrak{g}^{*}+\mathfrak{i}$ would be shown to be a Lie algebra and $\mathfrak{i}$ an ideal.

To see that $[\mathfrak{g}, \mathfrak{i}] \subseteq \mathfrak{i}$ we have only to show that for $x, y, z$ in $\mathfrak{g}$, $\left[x,\left[y, z^{*}\right]\right]$ is in $i$. Now

$$
\left[z,\left[y, z^{*}\right]\right]=\left[[z, y], z^{*}\right]+\left[y,\left[z, z^{*}\right]\right]=\left[[z, y], z^{*}\right] \in \mathfrak{i} .
$$

So $\left[x+z,\left[y,(x+z)^{*}\right]\right]$ is in $\mathrm{i}$. But

$$
\begin{aligned}
{\left[x+z,\left[y,(x+z)^{*}\right]\right]=} & {\left[x,\left[y, x^{*}\right]\right]+\left[x,\left[y, z^{*}\right]\right] } \\
& +\left[z,\left[y, x^{*}\right]\right]+\left[z,\left[y, z^{*}\right]\right] .
\end{aligned}
$$

The first and last terms are in $i$, so $\left[x,\left[y, z^{*}\right]\right]+\left[z,\left[y, x^{*}\right]\right] \in \mathfrak{i}$. We claim that $\left[z,\left[y, x^{*}\right]\right] \equiv\left[x,\left[y, z^{*}\right]\right](\bmod i)$. To see this note that

$$
\left[y, x^{*}\right]=\left[x, y^{*}\right]^{*}=-\left[x, y^{*}\right]=\left[y^{*}, x\right] \text {. }
$$

So we have

$$
\begin{aligned}
{\left[z,\left[y, x^{*}\right]\right] } & =\left[z,\left[y^{*}, x\right]\right]=\left[\left[z, y^{*}\right], x\right]+\left[y^{*},[z, x]\right] \\
& \equiv\left[\left[z, y^{*}\right], x\right](\bmod \mathfrak{i}) \equiv\left[\left[z^{*}, v\right], x\right](\bmod \mathfrak{i}) \\
& \equiv\left[x,\left[y, z^{*}\right]\right](\bmod \mathfrak{i})
\end{aligned}
$$

And we finally obtain

$2\left[x,\left[y, z^{*}\right]\right] \equiv\left(\left[x,\left[y, z^{*}\right]\right]+\left[z,\left[y, x^{*}\right]\right]\right)(\bmod \mathfrak{i}) \equiv 0(\bmod \mathfrak{i}) \quad$ Q.E.D.

We now assume that the ground field is of characteristic 0 . Then the finite-dimensional representations of a semisimple Lie algebra are completely reducible. (See for example [1, Theorem 8, p. 79].)

Theorem. Let $\mathfrak{g}$ be a semisimple subalgebra of $\mathfrak{u}$. Then for $x$ in $\mathfrak{g}$ we may uniquely write $x=x_{0}+x_{1}$ where $x_{0}$ is nrml and $x_{1}$ is skew. Moreover, for any $y$ in $\mathrm{g}$, if we similarly write $y=y_{0}+y_{1}$ then $\left[x_{0}, y_{1}\right]=\left[y_{0}, x_{1}\right]$ $=0$ and $\left[x_{0},\left(y_{0}\right)^{*}\right]=0$. Stated in other terms, the minimal self-adjoint subalgebra $\mathfrak{g}^{+}$of $\mathfrak{\mathfrak { H }}$ containing $\mathfrak{g}$ is the Lie algebra direct sum of three ideals, $\mathfrak{a}, \mathfrak{a}^{*}$, and $\mathfrak{i}$, with $\mathfrak{g} \subseteq \mathfrak{a}+\mathfrak{i}$ and $x^{*}=x$ for $x \in \mathfrak{i}$.

Proof. We will apply the fact that the finite-dimensional representations of $\mathfrak{g}$ are completely reducible to the adjoint representation of $\mathfrak{g}$ on ideals in $\mathfrak{g}^{+}=\mathfrak{g}+\mathfrak{g}^{*}+\mathfrak{i}, \mathfrak{i}=\left[\mathfrak{g}, \mathfrak{g}^{*}\right]$.

Let $g_{0}=\left\{x \in g^{+} \mid[x, \mathfrak{i}]=\{0\}\right\}$. We wish to make several observations about $g_{0}$. First, $g_{0}$ is an ideal in $\mathfrak{g}^{+}$. In fact it is the centralizer of the ideal $\mathfrak{i}$ in $\mathfrak{g}^{+}$and is therefore an ideal. 
Second, $\mathfrak{g}_{0}^{*}=g_{0}$. Indeed, if $x \in \mathfrak{g}_{0}$ then

$$
\left[x^{*}, \mathfrak{i}\right]=\left[x, \mathfrak{i}^{*}\right]^{*}=[x, \mathfrak{i}]^{*}=\{0\} .
$$

So $x^{*} \in g_{0}$. Since $x^{* *}=x$, we have $x \in g_{0}$ if and only if $x^{*} \in g_{0}$.

Third, $\mathfrak{g}_{0}+\mathfrak{i}=\mathfrak{g}^{+}$. To see this note that for any $x \in \mathfrak{g}^{+}$such that $x^{*}=x$, and any $y \in i$, we have

$$
-[x, y]=[x, y]^{*}=\left[y^{*}, x^{*}\right]=[-y, x]=[x, y] \text {. }
$$

So $[x, y]=0$. Thus $[x, \mathfrak{i}]=\{0\}$; so $x \in g_{0}$. If we pick $z \in \mathfrak{g}$ and let $x=z+z^{*}$ then $z+z^{*} \in g_{0}$. For any $y \in g$ we have then that $[y, z]$ $=\left[y, z+z^{*}\right]-\left[y, z^{*}\right]$. The first term is in $g_{0}$ since $g_{0}$ is an ideal; the second term is in $i$. So we have that $[\mathfrak{g}, \mathfrak{g}] \subseteq g_{0}+\mathfrak{i}$. Since $\mathfrak{g}$ is semisimple, $\mathfrak{g}=[\mathfrak{g}, \mathfrak{g}] \subseteq g_{0}+\mathfrak{i}$. Since $\mathfrak{g}_{0}^{*}=\mathfrak{g}_{0}$, we have that

$$
\mathfrak{g}^{*} \subseteq\left(\mathfrak{g}_{0}+\mathfrak{i}\right)^{*}=\left(\mathfrak{g}_{0}\right)^{*}+\mathfrak{i}^{*}=\mathfrak{g}_{0}+\mathfrak{i} .
$$

So

$$
\mathfrak{g}^{+}=\mathfrak{g}+\mathfrak{g}^{*}+\mathfrak{i} \subseteq \mathfrak{g}_{0}+\mathfrak{i} .
$$

Fourth, $g_{0} \cap i=\{0\}$. Let $\mathfrak{i}_{0}=\mathfrak{g}_{0} \cap \mathfrak{i}$. Then

$$
\begin{aligned}
& {\left[\mathfrak{g}_{0}, \mathfrak{i}_{0}\right] \subseteq\left[\mathfrak{g}_{0}, \mathfrak{i}\right]=\{0\}, \quad\left[\mathfrak{i}, \mathfrak{i}_{0}\right] \subseteq\left[\mathfrak{i}, \mathfrak{g}_{0}\right]=\{0\},} \\
& {\left[\mathfrak{g}^{+}, \mathfrak{i}_{0}\right] \subseteq\left[\mathfrak{g}_{0}+\mathfrak{i}, \mathfrak{i}_{0}\right] \subseteq\left[\mathfrak{g}_{0}, \mathfrak{i}_{0}\right]+\left[\mathfrak{i}, \mathfrak{i}_{0}\right]=\{0\} .}
\end{aligned}
$$

In particular, $\left[\mathfrak{g}, \mathfrak{i}_{0}\right]=\{0\}$. Since $\mathfrak{i}$ is completely reducible under the action of $\operatorname{ad}(\mathfrak{g})$, there is a linear compliment $\mathfrak{i}_{1}$ in $\mathfrak{i}$ to $\mathfrak{i}_{0}$ such that $\left[\mathfrak{g}, \mathfrak{i}_{1}\right] \subseteq \mathfrak{i}_{1}$. So

$$
[\mathfrak{g}, \mathfrak{i}]=\left[\mathfrak{g}, \mathfrak{i}_{0}+\mathfrak{i}_{1}\right] \subseteq\left[\mathfrak{g}, \mathfrak{i}_{0}\right]+\left[\mathfrak{g}, \mathfrak{i}_{1}\right] \subseteq\left[\mathfrak{g}, \mathfrak{t}_{1}\right] \subseteq \mathfrak{i}_{1}
$$

We may show however that $[\mathfrak{g}, \mathfrak{i}]=\mathfrak{i}$, proving $\mathfrak{i}_{1}=\mathfrak{i}$ and $\mathfrak{i}_{0}=\{0\}$. To see that $[g, \mathfrak{i}]=\mathfrak{i}$, consider the action of $\operatorname{ad}(\mathfrak{g})$ on $\mathfrak{g}^{*}+\mathfrak{i}$. Since $\mathfrak{g}^{*}+\mathfrak{i}$ is completely reducible under $\operatorname{ad}(\mathfrak{g})$, and $\left[\mathfrak{g}, \mathfrak{g}^{*}+\mathfrak{i}\right] \subseteq \mathfrak{i}$, we may find a complement $\mathfrak{i}^{\perp}$ in $\mathfrak{g}^{*}+\mathfrak{i}$ to $\mathfrak{i}$ such that $\left[\mathfrak{g}, \mathfrak{i}^{\perp}\right] \subseteq \mathfrak{i}^{\perp}$. But $\left[\mathfrak{g}, \mathfrak{i}^{\perp}\right] \subseteq \mathfrak{i}$ so $\left[\mathfrak{g}, \mathfrak{i}^{\perp}\right] \subseteq \mathfrak{i} \cap \mathfrak{i}^{\perp}=\{0\}$. Now

$$
\mathfrak{i}=\left[\mathfrak{g}, \mathfrak{g}^{*}\right] \subseteq[\mathfrak{g}, \mathfrak{i} \perp+\mathfrak{i}]=[\mathfrak{g}, \mathfrak{i}]+[\mathfrak{g}, \mathfrak{i} \perp]=[\mathfrak{g}, \mathfrak{i}] \subseteq \mathfrak{i} .
$$

So $\mathfrak{i}=[\mathfrak{g}, \mathfrak{i}]$. Consequently $\mathfrak{g}_{0} \cap \mathfrak{i}=\{0\}$.

We have expressed $\mathfrak{g}^{+}$as the direct sum of two ideals, $g_{0}$ and $\mathfrak{i}$. Thus for all $x, y \in \mathfrak{g}$ we may uniquely write $x=x_{0}+x_{1}$ and $y=y_{0}+y_{1}$ where $x_{0}, y_{0} \in g_{0}$ and $x_{1}, y_{1} \in \mathfrak{i}$. Clearly $\left[x_{0}, y_{1}\right]=\left[x_{1}, y_{0}\right]=0$. Also $\left[x_{0},\left(y_{0}\right)^{*}\right]=\left[\left(x-x_{1}\right),\left(y-y_{1}\right)^{*}\right] \equiv\left[x, y^{*}\right](\bmod \mathfrak{i}) \equiv 0(\bmod \mathfrak{i})$. But $\mathfrak{g}_{0}^{*}=\mathfrak{g}_{0}$ so $\left[x_{0},\left(y_{0}\right)^{*}\right] \subseteq \mathfrak{g}_{0} \cap \mathfrak{i}=\{0\}$. Q.E.D.

As an application of this theorem let $V$ be a complex vector space, not necessarily finite dimensional, with an inner product denoted 
$\langle$,$\rangle , so that V$ may be regarded as a dense subset of a Hilbert space. (In applications to analytic representations of Lie groups, $V$ would be the "analytic domain" of [2].) By "operator" on $V$ we will mean a linear operator defined everywhere. An operator $x$ on $V$ has an adjoint if there is an operator $y$ on $V$ such that for all $v, v^{\prime} \in V,\left\langle x(v), v^{\prime}\right\rangle$ $=\left\langle v, y\left(v^{\prime}\right)\right\rangle$. If $y$ exists it is unique and we write $x^{*}=y$. Let $\mathfrak{u}$ be the set of operators on $V$ having an adjoint. Then $\mathfrak{u}$ is a linear space and in fact a Lie algebra with the usual bracket. (The adjoint of $x+y$ is $x^{*}+y^{*}$ and the adjoint of $x y$ is $y^{*} x^{*}$.) $\mathfrak{u}$ is stable under the map $x \rightarrow x^{*}$ which is an anti-automorphism of order 2 . We therefore have the following corollaries of the Lemma and Theorem:

CoROLlaRy 1. Let $\mathrm{g}$ be a complex Lie algebra and $\pi$ a holomorphic representation of $\mathfrak{g}$ on $V$ by $n r m l$ operators in $\mathfrak{u}$. Then $\pi$ commutes with its contragredient.

Proof. That $\pi$ is holomorphic means simply that it is complex linear. Identify $\mathfrak{g}$ with its image under $\pi$ in $\mathfrak{u}$. By the Lemma we have that for all $x, y \in \mathfrak{g},\left[x, y^{*}\right]$ is skew. Now $i x$ is also in $\mathfrak{g}$, so $\left[i x, y^{*}\right]$ $=i\left[x, y^{*}\right]$ is also skew. What we must show is that $\left[x, y^{*}\right]=0$. But

$$
\begin{aligned}
i\left[x, y^{*}\right] & =-i\left(-\left[x, y^{*}\right]\right)=-i\left(\left[x, y^{*}\right]\right)^{*} \\
& =\left(i\left[x, y^{*}\right]\right)^{*}=-i\left[x, y^{*}\right]=0 .
\end{aligned}
$$

COROLlARY 2 . Let $\mathrm{g}$ be a real semisimple Lie algebra and $\pi$ a representation of $\mathfrak{g}$ on $V$ by $n r m l$ operators $i n \mathfrak{u}$. Then there are representations $\pi_{0}$ and $\pi_{1}$ of $\mathfrak{g}$ on $V$ by operators in $\mathfrak{u}$, such that $\pi_{0}$ commutes with its contragredient, $\pi_{1}$ is a representation by skew elements, $\pi_{0}$ commutes with $\pi_{1}$, and for all $x \in \mathfrak{g}, \pi(x)=\pi_{0}(x)+\pi_{1}(x)$.

Proof. Identify $\mathfrak{g}$ with its image under $\pi$ in $\mathfrak{u}$ and apply the Theorem. Q.E.D.

A similar result holds over any field of characteristic 0 if we replace the Hermitian inner product on $V$ by some nonsingular symmetric bilinear form.

Professor Segal pointed out the following application of Corollary 2:

CoROllaRy 3. Let $\mathfrak{g}$ be a real semisimple Lie algebra and $\pi$ a finitedimensional representation on a complex vector space by normal operators. Then $\pi$ is a representation by skew-adjoint operators.

Proof. We apply Corollary 2 with $V$ finite dimensional. Then $\mathfrak{u}$ is the Lie algebra of all complex linear operators, nrml means normal, and skew means skew-adjoint. For the decomposition $\pi=\pi_{0}+\pi_{1}$ we must show that $\pi_{0}=0$. To do this extend $\pi_{0}$ to a holomorphic repre- 
sentation of the complexification of $g$ by complex linearity, again denoting this representation $\pi_{0}$. Let $\pi_{-}$be defined by $\pi_{-}(x)=\pi_{0}(x)$ $-\pi_{0}(x)^{*}$ for all $x$ in the complexification of $\mathrm{g}$. Since the extended representation $\pi_{0}$ commutes with its contragredient, $\pi_{-}$is a representation by skew-adjoint operators of a complex semisimple Lie algebra. But it is well known that no such finite-dimensional representations exist. So for all $x$ in $g$ we have $0=\pi_{-}(x)=\pi_{0}(x)-\pi_{0}(x)^{*}$ or in other words, $\pi_{0}$ is a representation by self-adjoint operators. But since the bracket of two such operators is skew-adjoint and $[\mathfrak{g}, \mathfrak{g}]=\mathfrak{g}$ we must have that $\pi_{0}=0$. Q.E.D.

To complete the picture of finite-dimensional representations by normal operators we remark that if $\mathfrak{g}$ is a real solvable Lie algebra and $\pi$ a representation on a finite-dimensional complex vector space $V$ by normal operators then $\pi([\mathfrak{g}, \mathfrak{g}])=\{0\}$. Indeed by Lie's theorem we may find a basis of $V$ with respect to which $\pi$ is a representation by upper triangular matrices. The bracket of two such matrices is nilpotent so the image of $[\mathfrak{g}, \mathfrak{g}]$ under $\pi$ is a set of nilpotent normal matrices; but the only matrix with both these properties is 0 .

Finally, if $\mathfrak{g}$ is any real Lie algebra, write $\mathfrak{g}=\mathfrak{f}+\mathfrak{r}$ by the Levi decomposition, where $\mathfrak{l}$ is semisimple and $\mathfrak{r}$ is the maximal solvable ideal in g. Suppose $\pi$ is a faithful finite-dimensional representation by normal operators on a complex vector space. Then by the above remark, $\mathfrak{r}$ is abelian; by Corollary $3, \pi$ restricted to $\mathfrak{t}$ is skew-adjoint. The set $[\mathfrak{f}, \mathfrak{r}]$ is stable under the action of $\operatorname{ad}(\mathfrak{f})$ on $\mathfrak{r}$ and consequently has a linear compliment in $\mathfrak{r}$ stable under ad(t), namely the center of $\mathfrak{g}$. Now for $x \in \pi(\mathfrak{l}), y \in \pi(\mathfrak{r})$, we have $[x, y]=\left[x,\left(y-y^{*}\right)\right]+\left[x, y^{*}\right]$. The last term is skew-adjoint (by the Lemma) as is the first, so the operators of $\pi([\mathfrak{l}, \mathfrak{r}])$ are skew-adjoint. But a Lie algebra may have a finite-dimensional representation by skew-adjoint operators only if it is the direct sum of a compact semisimple Lie algebra and a central subalgebra. Applying this fact to $\mathfrak{f}+[\mathfrak{l}, \mathfrak{r}]$ we have $[\mathfrak{l},[\mathfrak{l}, \mathfrak{r}]]=0$. So all of $\mathfrak{r}$ commutes with $\mathfrak{l}$. Thus $\mathfrak{g}$ is the Lie algebra direct sum of a compact semisimple Lie algebra and an abelian Lie algebra. Thus the assumption that a finite-dimensional representation of a Lie algebra is by normal operators rather than skew-adjoint ones is no real increase of generality.

\section{BIBLIOGRAPHY}

1. Nathan Jacobson, Lie algebras, Wiley, New York, 1962.

2. I. E. Segal, Infinite-dimensional irreducible representations of compact semi-simple groups, Bull. Amer. Math. Soc. 70 (1964), 155-160.

Massachusetts Institute of TeChNology aNd The Institute for AdVANCEd StUdY 